\title{
Saúde mental e Covid-19: uma revisão integrativa da literatura
}

\author{
Mental health and Covid-19: an integrative literature review
}

Larissa Aline Carneiro Lobo', Carmen Esther Rieth ${ }^{1}$

DOI: $10.1590 / 0103-1104202113024$

RESUMO A pandemia do novo coronavírus (Covid-19) é uma realidade no Brasil e no mundo com potencial para desencadear sintomas psicológicos. O objetivo deste trabalho foi realizar uma revisão integrativa da literatura sobre saúde mental e pandemias. Foram cruzados os unitermos "saúde mental AND Covid-19 OR coronavírus OR pandemias AND intervenções em saúde mental OR psicotrópicos OR sintomatologia psiquiátrica OR psicopatologias OR fator de proteção em saúde mental" nas bases Scientific Electronic Library Online, Centro Latino-Americano e do Caribe de Informação em Ciências da Saúde e National Library of Medicine (PubMed), resultando em 1.642 artigos publicados em português, inglês e espanhol, no período de 2015 a 2020. Foram selecionados 43 artigos, de acordo com critérios de inclusão e exclusão, e todos foram publicados em 2020, referindo-se ao primeiro ano da pandemia de Covid-19. Sintomas depressivos e ansiosos, estresse e insônia foram frequentemente relatados e estiveram relacionados a ser profissional da saúde, adulto jovem, mulher; ter menor escolaridade e renda, histórico de doença crônica ou transtorno psiquiátrico e maior exposição nas mídias. Algumas intervenções psicológicas podem ser utilizadas para minimizar os impactos negativos da pandemia de Covid-19. Além disto, fatores de proteção podem ser explorados para reduzir os prejuízos na saúde mental dos indivíduos e das coletividades.

PALAVRAS-CHAVE Saúde mental. Covid-19. Coronavírus.

\begin{abstract}
The new coronavirus pandemic (Covid-19) is a reality in Brazil and worldwide, with potential to generate psychological symptoms. The aim of this study was to carry out an integrative literature review of mental health and pandemics. We used the crossing of the keywords: "mental health AND Covid-19 OR coronavirus OR pandemics AND mental health interventions OR psychotropics OR psychiatric symptoms OR psychopathologies OR protective factor in mental health" in Scientific Electronic Library Online, Latin American and Caribbean Center on Health Sciences Information and National Library of Medicine - PubMed databases, with 1.642 articles published in Portuguese, English and Spanish. After reading the titles, the preselection abstracts and following inclusion and exclusion criteria, 43 studies were included in this review. All articles were from 2020 and referred to the first year of the Covid-19 pandemic. Several reports of depression and anxiety symptoms, stress and insomnia were found and were related to being a health professional, young adult, woman, low level of education and income, history of chronic illness or psychiatric disorder and high media exposure. Some psychological interventions can minimize the negative impacts of the Covid-19 pandemic and protective factors can reduce damage to mental health of individuals and communities.
\end{abstract}

KEYWORDS Mental health. Covid-19. Coronavirus.

1 Universidade Feevale (Feevale) - Novo Hamburgo (RS), Brasil. lalilobo@yahoo.com.br 


\section{Introdução}

A pandemia do novo coronavírus (Covid-19) é uma realidade no Brasil e no mundo, e representa uma situação de crise com reflexos sociais, econômicos e na saúde das populações. Em janeiro de 2020, a Organização Mundial da Saúde (OMS) declarou como emergência de saúde pública o surto do novo coronavírus e, em março do mesmo ano, finalmente, classificou a Covid-19 como uma pandemia. Desde então, a OMS e organizações de saúde pública de todo o mundo vêm buscando conter sua disseminação'.

Pandemias como a de Covid-19 afetam pessoas no mundo inteiro, sem distinção de etnia, cultura, gênero ou nacionalidade, e momentos críticos como este têm grande potencial para desencadear sintomas psicológicos na população. Em situações de pandemia, os indivíduos sentem-se preocupados e estressados, especialmente as populações mais vulneráveis e os profissionais de serviços essenciais, como profissionais da saúde, comércio e manutenção, transporte e logística, segurança e defesa, entre outros, que garantem os serviços indispensáveis à comunidade. $\mathrm{O}$ medo, o estresse e as incertezas podem levar a consequências em longo prazo, acarretando problemas relacionados à saúde mental ${ }^{1}$.

Os problemas de saúde mental podem surgir em vista do temor da exposição e do contágio, do confinamento e do isolamento, e pelas medidas de quarentena, que implicam em mudanças na rotina de vida das pessoas².

As reações de medo e as preocupações da população podem ser acentuadas pelo excesso de informações, muitas vezes oriundas de fontes não confiáveis, pelas fake news e especulações da mídia e redes sociais. Dispor de informações fidedignas, transparentes e oportunas é vital para a adequada saúde mental da população ${ }^{3,4}$.

O objetivo deste trabalho foi realizar uma revisão integrativa da literatura sobre saúde mental e pandemias. Esta proposta se deu em virtude do pouco conhecimento sobre o impacto de uma pandemia no adoecimento mental, em meio ao cenário da Covid-19, no primeiro ano da pandemia. Considerou-se relevante investigar quais as principais psicopatologias/sintomas psiquiátricos que se manifestam, de que forma estas reações podem ser contornadas, que fatores protegem o indivíduo do adoecimento mental e que intervenções psicoterapêuticas e psicofarmacológicas têm sido utilizadas.

\section{Metodologia}

\section{Delineamento do estudo}

O presente estudo consiste em uma revisão integrativa da literatura. O objetivo desta metodologia é obter um conhecimento profundo de determinado fenômeno, baseando-se em estudos anteriores sobre o tema. Este método permite que as pesquisas publicadas sejam sintetizadas em um único artigo, tornando os resultados mais acessíveis. Estudos deste tipo devem ser conduzidos através de critérios metodológicos rigorosos, seguindo etapas bem descritas e com resultados apresentados com clareza ${ }^{5,6}$. Foram consultadas as bases de dados Scientific Electronic Library Online (SciELO) (https://scielo.org/); Centro LatinoAmericano e do Caribe de Informação em Ciências da Saúde (Bireme)/Organização PanAmericana da Saúde (Opas)/OMS (https:// bvsalud.org/); e National Library of Medicine (NLM) - PubMed (https://www.ncbi.nlm. nih.gov/pubmed/). Foram incluídos artigos publicados entre os anos de 2015 e 2020, nos idiomas espanhol, inglês e português. Para a estratégia de busca foi utilizada a combinação dos unitermos "saúde mental AND Covid-19 OR coronavírus OR pandemias AND intervenções em saúde mental OR psicotrópicos OR sintomatologia psiquiátrica OR psicopatologias OR fator de proteção em saúde mental”.

Para a extração dos dados, tomou-se como base o formato utilizado por Ursi e Galvão7, 
que prevê os seguintes itens: identificação do artigo original, características metodológicas e avaliação do rigor do estudo, das intervenções mensuradas e dos resultados encontrados.

Dessa forma, para a seleção dos estudos foram seguidas as seguintes etapas: leitura dos títulos de todos os artigos encontrados; leitura dos resumos da pré-seleção, de acordo com critérios de inclusão e exclusão; leitura, na íntegra, dos artigos da amostra parcial; exploração dos artigos; codificação dos conteúdos emergentes e relevantes; e apresentação dos resultados a partir de categorias identificadas no material pesquisado 7 .

\section{Descrição dos critérios de inclusão, exclusão e seleção dos estudos}

A busca nas três bases de dados obteve 1.642 resultados, sendo 1.615 artigos da base Bireme, 21 da SciELO e 6 da PubMed. Após a leitura dos 1.642 títulos, foram pré-selecionados 622 artigos, cujos resumos foram lidos. Excluíramse os artigos duplicados, os do tipo letter/carta ao editor/editorial/opinião, os artigos no prelo/ preprint, artigos incompletos, indisponíveis ao acesso, documentos cujo formato não se enquadrava como artigo, artigos de revisão e os que não atendiam ao objetivo deste estudo. Após as exclusões, restaram 150 artigos para leitura integral, seguindo-se os mesmos critérios de exclusão acima descritos. Ao final, foram selecionados 43 artigos para compor esta revisão.

Os artigos selecionados foram organizados em duas categorias principais: profissionais da saúde e grandes populações/países. Posteriormente, foram apresentados conforme as seguintes temáticas: Sintomatologia psiquiátrica/Psicopatologias: Presença de sintomas psiquiátricos e/ou psicopatologias em profissionais da saúde, Presença de sintomas psiquiátricos e/ou psicopatologias em grandes populações; Fatores associados à presença de sintomas psiquiátricos e psicopatologias; Fatores de proteção em saúde mental; e Intervenções em saúde mental: intervenções em saúde mental para os profissionais da saúde e Intervenções em saúde mental para a população geral.

\section{Resultados}

\section{Considerações sobre a amostra}

É importante ressaltar que foi empregado, na estratégia de busca, o unitermo "pandemias", e o período analisado compreendeu os anos de 2015 a 2020, a fim de localizar artigos que relacionassem saúde mental com experiências de epidemias anteriores, contudo, todos os artigos localizados nesta revisão integrativa foram publicados em 2020 e referiam-se ao primeiro ano da pandemia de Covid-19. Além disso, a busca pelo unitermo "psicofármacos" não localizou nenhum artigo. O detalhamento dos critérios de inclusão e exclusão dos artigos está descrito na figura 1. 
Figura 1. Diagrama dos artigos incluídos e excluídos, de acordo com critérios de inclusão e exclusão estabelecidos no estudo

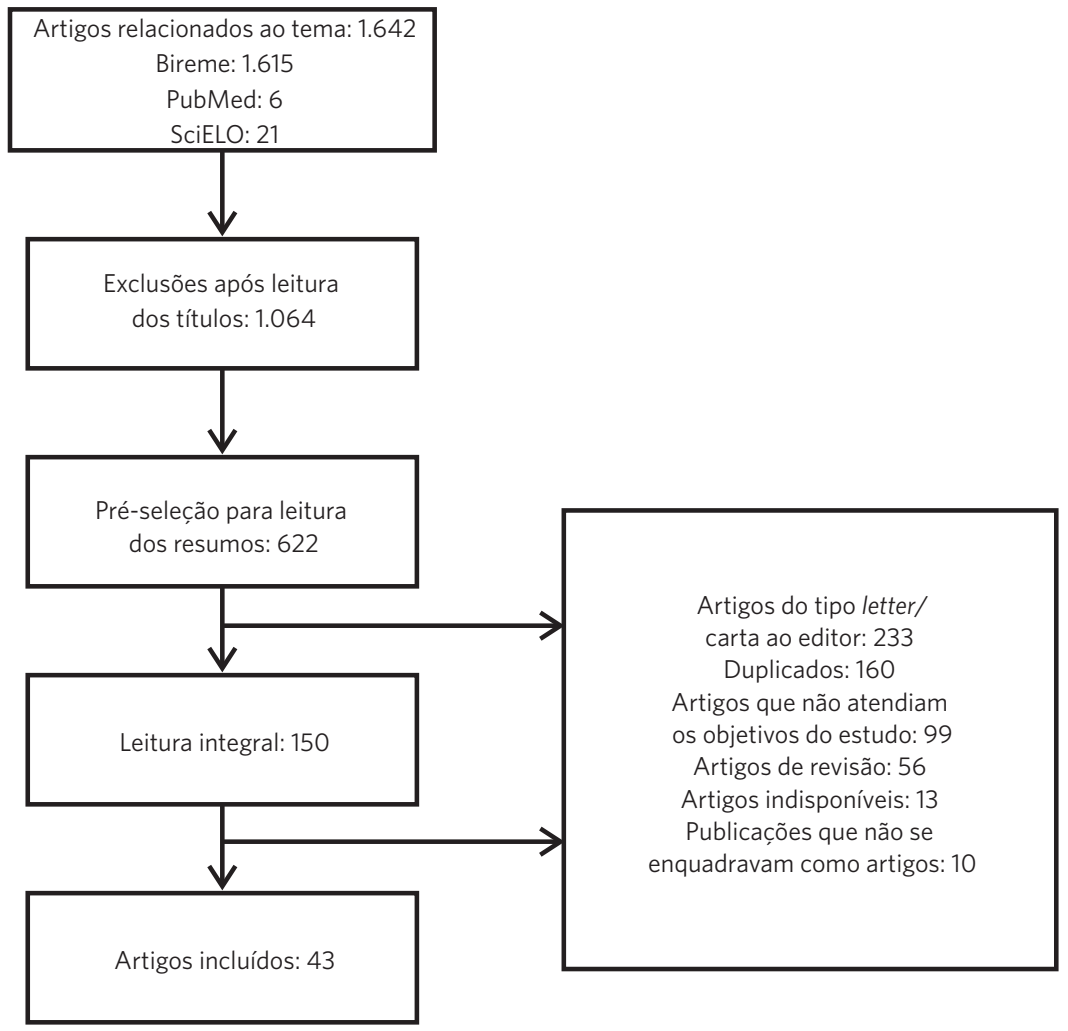

Fonte: Elaboração própria

A amostra deste estudo foi composta por 43 artigos originais, publicados em 2020 e provenientes de 19 países, conforme ilustra o quadro 1. Das 43 pesquisas realizadas, 19 foram conduzidas na China. Dos 43 estudos,
40 estavam redigidos em língua inglesa e 3 em língua portuguesa. O delineamento dos estudos conforme definido pelos autores foi transversal (com questionário on-line) para 42 artigos e caso-controle para 1 artigo.

Quadro 1. Distribuição geral dos artigos selecionados.

\begin{tabular}{|c|c|c|c|c|c|}
\hline $\mathbf{n}$ & Autor & Ano & País & Delineamento & Público-alvo \\
\hline A1 & Cai Q et al. & 2020 & China & Caso-controle & Médicos \\
\hline A2 & Kannampallil TG et al. & 2020 & Estados Unidos & Transversal & Médicos \\
\hline A3 & Li X et al. & 2020 & China & Transversal & População geral \\
\hline A4 & Si MY et al. & 2020 & China & Transversal & Funcionários hospital \\
\hline A5 & Sljivo A et al. & 2020 & Bósnia e Herzegovina & Transversal & População geral \\
\hline A6 & Moreno LL et al. & 2020 & Espanha & Transversal & Profissional da saúde \\
\hline A7 & Rodriguez LM et al. & 2020 & Estados Unidos & Transversal & População geral \\
\hline A8 & Shi L et al. & 2020 & China & Transversal & População geral \\
\hline
\end{tabular}




\begin{tabular}{|c|c|c|c|c|c|}
\hline \multicolumn{6}{|c|}{ Quadro 1. (cont.) } \\
\hline $\mathrm{n}$ & Autor & Ano & País & Delineamento & Público-alvo \\
\hline A9 & Gualano MR et al. & 2020 & Itália & Transversal & População geral \\
\hline A10 & Duan L et al. & 2020 & China & Transversal & Estudantes \\
\hline A11 & Barros MBA et al. & 2020 & Brasil & Transversal & População geral \\
\hline $\mathrm{A} 12$ & Salgado JG et al. & 2020 & Espanha & Transversal & População geral \\
\hline A13 & Stanton $\mathrm{R}$ et al. & 2020 & Austrália & Transversal & População geral \\
\hline $\mathrm{A} 14$ & Kim YJ e Cho JH. & 2020 & Coreia do Sul & Transversal & População geral \\
\hline A15 & Guo J et al. & 2020 & China & Transversal & População geral \\
\hline A16 & Antunes R et al. & 2020 & Portugal & Transversal & População geral \\
\hline A17 & Temsah MH et al. & 2020 & Arábia Saudita & Transversal & Profissional da saúde \\
\hline A18 & Zerbini G et al. & 2020 & Alemanha & Transversal & Médicos \\
\hline A19 & Man MA et al. & 2020 & Romênia & Transversal & Profissional da saúde \\
\hline A20 & Solomou I et al. & 2020 & Chipre & Transversal & População geral \\
\hline A21 & Xiao X et al. & 2020 & China & Transversal & Profissional da saúde \\
\hline A22 & Guo Y et al. & 2020 & China & Transversal & População geral \\
\hline A23 & Taylor S et al. & 2020 & Canadá/Estados Unidos & Transversal & População geral \\
\hline A24 & Duarte MQ et al. & 2020 & Brasil & Transversal & População geral \\
\hline A25 & Zhou SJ et al. & 2020 & China & Transversal & Estudantes \\
\hline A26 & O'Moore K et al. & 2020 & Austrália & Transversal & População geral \\
\hline A27 & Pedrozo-Pupo JC et al. & 2020 & Colômbia & Transversal & População geral \\
\hline A28 & Naser AY et al. & 2020 & Jordânia & Transversal & População geral \\
\hline A29 & Dal'Bosco E et al. & 2020 & Brasil & Transversal & Profissional da saúde \\
\hline A30 & Ying $Y$ et al. & 2020 & China & Transversal & Familiares \\
\hline A31 & Chao M et al. & 2020 & China & Transversal & População geral \\
\hline A32 & Kowitt SD et al. & 2020 & Estados Unidos & Transversal & População geral \\
\hline A33 & Özdin S e Bayrak ÖȘ. & 2020 & Turquia & Transversal & População geral \\
\hline A34 & El-Zoghby SM et al. & 2020 & Egito & Transversal & População geral \\
\hline A35 & Roy D et al. & 2020 & Índia & Transversal & População geral \\
\hline A36 & Mazza C et al. & 2020 & Itália & Transversal & População geral \\
\hline A37 & Tang W et al. & 2020 & China & Transversal & Universitários \\
\hline A38 & Wang C et al. & 2020 & China & Transversal & População geral \\
\hline A39 & Huang Y e Zhao N. & 2020 & China & Transversal & População geral \\
\hline A40 & Tian F et al. & 2020 & China & Transversal & População geral \\
\hline A41 & Zhang WR et al. & 2020 & China & Transversal & População geral \\
\hline A42 & Lai J et al. & 2020 & China & Transversal & Médicos \\
\hline A43 & Ahmed MZ et al. & 2020 & China & Transversal & População geral \\
\hline
\end{tabular}

Fonte: Elaboração própria. 
Quanto ao tipo de população analisada, 29 estudos avaliaram os efeitos da pandemia de Covid-19 na saúde mental de grandes populações/países ${ }^{8-36}$. O efeito da pandemia de Covid-19 na saúde mental dos profissionais da saúde foi analisado em dez artigos ${ }^{37-46}$ e um artigo avaliou a saúde mental de familiares dos profissionais da saúde ${ }^{47}$. Outra população de interesse dos estudos foi a composta por estudantes, tendo crianças e adolescentes sido avaliados em dois artigos ${ }^{\mathbf{4 8 , 4 9}} \mathrm{e}$ um artigo enfocado estudantes universitários ${ }^{50}$. A distribuição dos artigos conforme categoria de interesse está descrita nos quadros 2 e 3.

\begin{tabular}{|c|c|}
\hline Autor & Título \\
\hline Stanton R et al. & $\begin{array}{l}\text { Depression, Anxiety and Stress during COVID-19: Associations with Changes in Physical Activity, Sleep, Tobacco and Alco- } \\
\text { hol Use in Australian Adults. }\end{array}$ \\
\hline O'Moore K et al. & Acute mental health responses during the COVID-19 pandemic in Australia. \\
\hline Duarte MQ et al. & COVID-19 e os impactos na saúde mental: uma amostra do Rio Grande do Sul, Brasil. \\
\hline Barros MBA et al. & $\begin{array}{l}\text { Relato de tristeza/depressão, nervosismo/ansiedade e problemas de sono na população adulta brasileira durante a pande- } \\
\text { mia de COVID-19. }\end{array}$ \\
\hline Sljivo A et al. & Fear and Depression among Residents of Bosnia and Herzegovina during COVID-19 Outbreak - Internet Survey. \\
\hline Taylor S et al. & COVID stress syndrome: Concept, structure, and correlates. \\
\hline Wang C et al. & A longitudinal study on the mental health of general population during the COVID-19 epidemic in China. \\
\hline Huang Y e Zhao N. & $\begin{array}{l}\text { Generalized anxiety disorder, depressive symptoms and sleep quality during COVID-19 outbreak in China: a web-based } \\
\text { cross-sectional survey. }\end{array}$ \\
\hline Tian F et al. & Psychological symptoms of ordinary Chinese citizens based on SCL-90 during the level I emergency response to COVID-19. \\
\hline Zhang WR et al. & Mental Health and Psychosocial Problems of Medical Health Workers during the COVID-19 Epidemic in China. \\
\hline Ahmed MZ et al. & Epidemic of COVID-19 in China and associated Psychological Problems. \\
\hline Li X et al. & Factors Associated with Mental Health Results among Workers with Income Losses Exposed to COVID-19 in China. \\
\hline Shi L et al. & $\begin{array}{l}\text { Prevalence of and Risk Factors Associated With Mental Health Symptoms Among the General Population in China During } \\
\text { the Coronavirus Disease } 2019 \text { Pandemic. }\end{array}$ \\
\hline Guo J et al. & $\begin{array}{l}\text { Coping with COVID-19: Exposure to COVID-19 and Negative Impact on Livelihood Predict Elevated Mental Health Problems } \\
\text { in Chinese Adults. }\end{array}$ \\
\hline Chao M et al. & Media use and acute psychological outcomes during COVID-19 outbreak in China. \\
\hline Guo Y et al. & $\begin{array}{l}\text { Disorders and Associated Risk Factors in Quarantined Adults During the COVID-19 Outbreak in China: Cross-Sectional } \\
\text { Study. }\end{array}$ \\
\hline Solomou l et al. & $\begin{array}{l}\text { Prevalence and Predictors of Anxiety and Depression Symptoms during the COVID-19 Pandemic and Compliance with } \\
\text { Precautionary Measures: Age and Sex Matter. }\end{array}$ \\
\hline Pedrozo-Pupo JC et al. & Perceived stress associated with COVID-19 epidemic in Colombia: an online survey. \\
\hline Kim YJ e Cho JH. & $\begin{array}{l}\text { Correlation between Preventive Health Behaviors and Psycho-Social Health Based on the Leisure Activities of South Koreans } \\
\text { in the COVID-19 Crisis. }\end{array}$ \\
\hline El-Zoghby SM et al. & Impact of the COVID-19 Pandemic On Mental Health and Social Support Among Adult Egyptians. \\
\hline Salgado JG et al. & Related Health Factors of Psychological Distress During the COVID-19 Pandemic in Spain. \\
\hline Kowitt SD et al. & Tobacco Quit Intentions and Behaviors among Cigar Smokers in the United States in Response to COVID-19. \\
\hline Rodriguez LM et al. & $\begin{array}{l}\text { Drinking to cope with the pandemic: The unique associations of COVID-19 - related perceived threat and psychological } \\
\text { distress to drinking behaviors in American men and women. }\end{array}$ \\
\hline Gualano MR et al. & Effects of Covid-19 Lockdown on Mental Health and Sleep Disturbances in Italy. \\
\hline Mazza C et al. & $\begin{array}{l}\text { A Nationwide Survey of Psychological Distress among Italian People during the COVID-19 Pandemic: Immediate Psycholo- } \\
\text { gical Responses and Associated Factor. }\end{array}$ \\
\hline Roy D et al. & Study of knowledge, attitude, anxiety \& perceived mental healthcare need in Indian population during COVID-19 pandemic. \\
\hline
\end{tabular}




\begin{tabular}{|c|c|}
\hline Autor & Título \\
\hline Naser AY et al. & $\begin{array}{l}\text { Mental health status of the general population, healthcare professionals, and university students during } 2019 \text { coronavirus } \\
\text { disease outbreak in Jordan: A cross-sectional study. }\end{array}$ \\
\hline Roy D et al. & $\begin{array}{l}\text { Exploring Lifestyle Habits, Physical Activity, Anxiety and Basic Psychological Needs in a Sample of Portuguese Adults during } \\
\text { COVID-19. }\end{array}$ \\
\hline Özdin S e Bayrak Öș. & $\begin{array}{l}\text { Levels and predictors of anxiety, depression and health anxiety during COVID-19 pandemic in Turkish society: The impor- } \\
\text { tance of gender. }\end{array}$ \\
\hline Duan L et al. & An investigation of mental health status of children and adolescents in China during the outbreak of COVID-19. \\
\hline Zhou SJ et al. & $\begin{array}{l}\text { Prevalence and socio-demographic correlates of psychological health problems in Chinese adolescents during the outbreak } \\
\text { of COVID-19. }\end{array}$ \\
\hline Tang W et al. & $\begin{array}{l}\text { Prevalence and correlates of PTSD and depressive symptoms one month after the outbreak of the COVID-19 epidemic in a } \\
\text { sample of home-quarantined Chinese university students. }\end{array}$ \\
\hline
\end{tabular}

Fonte: Elaboração própria.

Quadro 3. Distribuição de artigos com foco em profissionais da saúde.

\begin{tabular}{|c|c|}
\hline Autor & Título \\
\hline Dal'Bosco E et al. & A saúde mental da enfermagem no enfrentamento da COVID-19 em um hospital universitário regional. \\
\hline Zerbini G et al. & $\begin{array}{l}\text { Psychosocial burden of healthcare professionals in times of COVID-19 - a survey conducted at the University Hospital Au- } \\
\text { gsburg. }\end{array}$ \\
\hline Temsah MH et al & The psychological impact of COVID-19 pandemic on health care workers in a MERS-CoV endemic country. \\
\hline Ying Y et al. & $\begin{array}{l}\text { Mental health status among family members of health care workers in Ningbo, China, during the coronavirus disease } 2019 \\
\text { (COVID-19) outbreak: a cross-sectional study. }\end{array}$ \\
\hline Cai Q et al. & $\begin{array}{l}\text { The mental health of frontline and non-frontline medical workers during the coronavirus disease } 2019 \text { (COVID-19) outbreak } \\
\text { in China: A case-control study. }\end{array}$ \\
\hline Xiao X et al. & $\begin{array}{l}\text { Psychological impact of healthcare workers in China during COVID-19 pneumonia epidemic: A multi-center cross-sectional } \\
\text { survey investigation. }\end{array}$ \\
\hline Si MY et al. & Psychological impact of COVID-19 on medical care workers in China. \\
\hline Lai J et al. & Factors Associated With Mental Health Outcomes Among Health Care Workers Exposed to Coronavirus Disease 2019. \\
\hline Kannampallil TG et al. & Exposure to COVID-19 patients increases physician trainee stress and burnout. \\
\hline Moreno LL et al. & $\begin{array}{l}\text { Symptoms of Posttraumatic Stress, Anxiety, Depression, Levels of Resilience and Burnout in Spanish Health Personnel du- } \\
\text { ring the COVID-19 Pandemic. }\end{array}$ \\
\hline Man MA et al. & Perception and Coping with Emotional Distress During COVID-19 Pandemic: A Survey Among Medical Staff. \\
\hline
\end{tabular}

Fonte: Elaboração própria.

A seguir, serão apresentados os principais achados do trabalho, relacionados ao objetivo estabelecido para esta revisão.

\section{Sintomatologia psiquiátrica/ psicopatologias}

Houve a identificação de pelo menos um sintoma psiquiátrico ou uma psicopatologia em cada um dos estudos que compuseram esta revisão. Ressalta-se que foram utilizados diferentes instrumentos para avaliar a presença de sintomas/psicopatologias nos diferentes países, em múltiplos períodos da pandemia. Além disso, o termo utilizado para descrever o sofrimento psicológico nas populações variou entre os estudos.

Os sintomas psiquiátricos ou as psicopatologias mais frequentemente relatadas nas diferentes populações e nos países analisados 
foram ansiedade, depressão, estresse e insônia, considerando como foco principal os profissionais da saúde e as grandes populações.

\section{Presença de sintomas psiquiátricos e/ou psicopatologias em profissionais da saúde}

Estudos analisaram o impacto psicológico sofrido pelos profissionais da saúde que atuavam na linha de frente do combate à covid19, comparado ao daqueles que não atendiam diretamente a esses pacientes ${ }^{19,37,38,42,43}$. Dois estudos chineses ${ }^{37,46}$ e um americano ${ }^{38}$ encontraram maior impacto psicológico em profissionais médicos que atuavam na linha de frente, quando comparados àqueles que não atendiam diretamente os pacientes com Covid-1946. Os profissionais da linha de frente apresentaram maiores sintomas de ansiedade, insônia, humor deprimido, estresse e burnout. Já estudos realizados na Romênia ${ }^{43}$ e na Alemanha ${ }^{\mathbf{4 2}}$ não encontraram diferenças significativas nas prevalências de sintomas psiquiátricos entre as equipes médicas. Maior presença de sintomas depressivos, ansiedade, exaustão, altos níveis de estresse e menor realização no trabalho foi verificada em profissionais da enfermagem que atuavam na linha de frente na Alemanha ${ }^{42}$, no Brasilis e na China ${ }^{46}$.

As prevalências de sintomas nos profissionais da saúde chineses foram superiores às encontradas na população geral ${ }^{33-35,39}$, sendo maiores entre profissionais médicos ${ }^{33-35,40}$. Na Jordânia ${ }^{25}$, altas prevalências de sintomas psiquiátricos foram verificadas em profissionais da saúde, sendo os níveis ainda maiores entre médicos pneumologistas. Os fatores associados ao maior impacto psicológico nesses profissionais foram: esgotamento, isolamento da família, sentimento de desamparo, escassez de Equipamentos de Proteção Individual (EPIs) e aumento do número de casos de Covid-19. Na província de Hubei, China, 97,9\% dos profissionais da saúde apresentaram pelo menos um sintoma de estresse pós-traumático. Aqueles que tiveram casos de Covid-19 em suas comunidades e eram enfermeiros mostraram-se mais propensos a ficar ansiosos ${ }^{39}$.

Profissionais da saúde da China ${ }^{39}$, Arábia Saudita ${ }^{\mathbf{4 1}}$ e Espanha ${ }^{\mathbf{4 0}}$ sentiam-se correndo alto risco de serem infectados e relataram preocupação com o contágio de seus familiares e amigos. Maior prevalência de sintomas esteve associada ao número de horas de plantão trabalhadas e à falta de EPIs adequados ${ }^{39,40}$. Estudo realizado com familiares de funcionários de cinco hospitais da China ${ }^{\mathbf{4 7}}$ encontrou altas prevalências de sintomas ansiosos (33,7\%) e depressivos (29,3\%) nesta população. A presença de sintomas nos familiares foi superior à encontrada na população geral da China, e inferior à dos profissionais médicos.

A presença de sintomas psiquiátricos e psicopatologias em profissionais da saúde durante a pandemia de Covid-19 foi superior à verificada em surtos anteriores, como na Síndrome Respiratória Aguda Grave (Sars) da China ${ }^{40}$, na Síndrome Respiratória do Oriente Médio (Mers-CoV) e na influenza sazonal da Arábia Saudita ${ }^{41}$.

\section{Presença de sintomas psiquiátricos e/ou psicopatologias em grandes populações}

Quanto ao impacto da pandemia de Covid-19 na situação de saúde das populações, estudo realizado nos Estados Unidos e no Canadá sugere que esta doença tem maior potencial para causar danos à saúde mental dos indivíduos, se comparada à saúde física. No estudo, apenas $2 \%$ dos entrevistados haviam sido diagnosticados com Covid-19 e 6\% conheciam alguém que havia recebido diagnóstico. Mesmo assim, 38\% da população estudada apresentaram algum nível de angústia ${ }^{27}$.

A preocupação com o risco de contrair a Covid-19 foi relatada em estudos realizados na Jordânia ${ }^{25}$ (78\%), Índia ${ }^{30}$ (37,8\%) e Austrália ${ }^{23}$ (25,9\%). Na Jordânia 25 (75\%) e na Austrália 23 (52,7\%), os entrevistados relataram estar preocupados com a contaminação de familiares e amigos. Na Bósnia e Herzegovina, mais da 
metade da população (53\%) relatou ter medo de morrer por Covid-19 e 11,2\% apresentaram ideação suicida ou automutilação 9 .

$\mathrm{Na}$ Austrália ${ }^{23}$, a população apresentou níveis elevados de estresse (64\%) e metade dos entrevistados relatou apresentar incerteza e solidão durante a pandemia de Covid-19. Elevação dos níveis de estresse também foi relatada por $15 \%$ da população da Colômbia ${ }^{24}$. No Egito $^{29}$, houve aumento do estresse doméstico (62,7\%), do financeiro (55,7\%) e do relacionado ao trabalho $(34,1 \%)$. Os egípcios relataram sentir-se horrorizados (53,9\%), impotentes $(52 \%)$ e apreensivos (66,3\%) diante da doença.

Altas prevalências de sintomas depressivos foram verificadas em diferentes países durante a pandemia de Covid-19: Itália ${ }^{31}$ (67,3\%), Austrália23 (62\%), Chipre ${ }^{19}(48,1 \%)$, Brasil $^{13}(40,4 \%)$, China $^{11}$ (27,9\%), Jordânia ${ }^{25}$ (23,8\%) e Turquia ${ }^{28}$ (23,6\%). Em relação a sintomas de ansiedade, níveis elevados foram verificados no Brasil13 (52,6\%), na Austrália 23 (50\%), na Turquia28 (45,1\%), no Chipre $^{19}$ (41\%), na China ${ }^{11}$ (31,6\%), na Itália ${ }^{12}$ $(23,2 \%)$ e na Jordânia 25 (13,1\%).

Com relação ao sono, em estudo realizado no Brasil13, $43,5 \%$ dos entrevistados relataram ter problemas para dormir e $48 \%$ tiveram o agravamento de problemas de sono pré-existentes. Distúrbios do sono também foram verificados nas populações italiana $(42,2 \%)^{\mathbf{1 2}}$, chinesa $(29,2 \%)^{11}$ e indiana $(12,5 \%)^{3}$.

\section{Fatores associados à presença de sintomas psiquiátricos $\mathrm{e}$ psicopatologias}

A maioria dos artigos verificou associações entre fatores sociodemográficos e pior saúde mental, tais como: gênero, local de residência, nível de

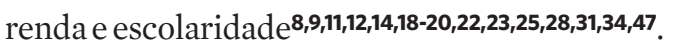
Outros fatores, como exposição à mídia e aos hábitos de vida, também foram relacionados ${ }^{10,14-16,19,20,26,27,33,36,48}$.

Maior prevalência de sintomas psicológicos/psicopatologias esteve associada ao gênero feminino na maioria dos estudos, em diferentes países $9,11,12,14,18,19,23,25,28,29,31,34,47$.
$\mathrm{O}$ fato de residir em zona urbana esteve relacionado a maiores níveis de medo na Bósnia e Herzegovina 9 , e maiores prevalências de sintomas psiquiátricos ocorreram na Turquia ${ }^{28}$ e no Egito 29 .

Em relação à situação conjugal, estudos realizados no Egito $^{29}$ e na Itália ${ }^{12}$ associaram o estado civil casado ou coabitado a um fator preditivo para uma pior saúde mental. $\mathrm{Na}$ $\mathrm{China}^{34}$, a presença de sintomas psicológicos foi relacionada a ser divorciado ou viúvo. Já na Bósnia e Herzegovina ${ }^{9}$, maiores níveis de medo foram relatados nos indivíduos solteiros.

Residir com outras pessoas no domicílio relacionou-se a uma pior saúde mental em estudo realizado no Chipre ${ }^{19}$. Já na China ${ }^{20}$, indivíduos que moravam sozinhos e aqueles que vivenciavam conflitos familiares apresentaram mais sintomas depressivos e ansiosos. Indivíduos sem filhos tiveram sua saúde mental mais prejudicada na Bósnia e Herzegovina ${ }^{9}$ e na Itália ${ }^{31}$.

As finanças pessoais, durante a pandemia de Covid-19, foram motivos de preocupação para as populações do Chipre ${ }^{19}$ e da Austrália ${ }^{23}$. No Brasil $^{\mathbf{2 2}}$, pessoas que sofreram perdas de renda relataram uma pior saúde mental. Na China, três diferentes estudos verificaram associação entre perdas de renda e maiores sintomas de depressão, ansiedade e insônia ${ }^{\mathbf{8}, 20,47}$. $\mathrm{Na}$ Espanha ${ }^{14}$, indivíduos de baixa renda apresentaram altos níveis de sofrimento psíquico. De maneira inversa, na Jordânia ${ }^{25}$, ter renda alta foi um fator associado à presença de sintomas psicopatológicos.

Sintomas psicológicos/psicopatologias foram mais prevalentes em desempregados na Bósnia e Herzegovina ${ }^{9}$, na Itália ${ }^{31}$ e no Chipre $^{19}$. Na Espanha ${ }^{14}$ e na Itália ${ }^{31}$, níveis elevados de angústia foram verificados em pessoas que trabalhavam fora de casa.

Adultos jovens apresentaram pior saúde

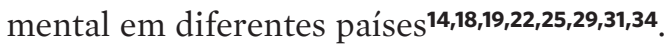
$\mathrm{O}$ achado relacionado à idade foi bastante consistente entre os estudos, divergindo apenas no sintoma de medo na Bósnia e Herzegovina. Segundo os autores, o medo 
estaria relacionado à experiência prévia de uma guerra nesta geração. Além disto, as manifestações da Covid-19 nas faixas etárias mais elevadas costumam ter desfechos mais graves?

Estudantes apresentaram maior sofrimento psicológico na Austrália ${ }^{23}$ e maior prevalência de sintomas depressivos na Bósnia e Herzegovina ${ }^{9}$, Jordânia ${ }^{25}$ e Itália ${ }^{31}$. Ser estudante universitário associou-se a uma maior prevalência de sintomas psicológicos no Egito ${ }^{29}$ e na Jordânia ${ }^{25}$. Maiores níveis de depressão e ansiedade entre os jovens estudantes estariam relacionados às mudanças na modalidade de ensino, preocupações com a formação e ao desempenho acadêmico ${ }^{25}$.

No Brasil22 e na Colômbia ${ }^{24}$, o acesso a informações imprecisas e orientações inconsistentes repassadas pelas autoridades sanitárias foi relacionado a maiores níveis de estresse. $\mathrm{Na}$ China, o ato de procurar constantemente por informações sobre a Covid-19 e o tempo de exposição às mídias relacionaram-se a efeitos psicológicos mais negativos ${ }^{26,33}$.

$\mathrm{O}$ efeito psicológico negativo associado ao uso das mídias foi mais evidente entre os jovens. Este público costuma utilizar tais recursos com maior frequência, assim obtendo maior volume de informações sobre a Covid19, nem sempre fidedignas ${ }^{20}$. Pessoas mais jovens ( $<35$ anos) e que passavam mais de três horas por dia buscando informações sobre a Covid-19 apresentaram maior prevalência de transtorno de ansiedade generalizada $(35,1 \%)$, sintomas depressivos $(20,1 \%)$ e pior qualidade de sono $(18,2 \%)^{33}$.

$\mathrm{Na}$ Índia ${ }^{30}, 36,4 \%$ da população relataram sintomas de estresse relacionado ao uso das mídias sociais. Na Itália' ${ }^{12}$ o tempo gasto na internet associou-se a uma maior presença de sintomas psiquiátricos. A associação entre o uso de mídias sociais e a presença de sintomas psicológicos, como ansiedade e depressão, foi encontrada em dois estudos chineses ${ }^{20,26}$ e em um indiano ${ }^{30}$.

Na Espanha ${ }^{14}$, foi verificado alto nível de sofrimento psíquico (72\%) em indivíduos que haviam tido contato com pacientes Covid-19, apresentado sintomas ou recebido diagnóstico de Covid-19. Na Bósnia e Herzegovina9 e na Itália ${ }^{29}$, indivíduos que conheciam alguém que havia sido contaminado pela Covid-19 tiveram piores respostas psicológicas ${ }^{31}$. Na China $^{20}$, pessoas com maior preocupação em relação à Covid-19, tanto em relação a si como aos seus familiares, tiveram maior presença de sintomas depressivos e ansiosos.

Estudo chinês" realizado na província de Hubei observou que $50 \%$ dos familiares ou amigos de pacientes com Covid-19 apresentaram sintomas psiquiátricos. Já entre os pacientes com diagnóstico de Covid-19, 75\% apresentaram sintomas depressivos e $71 \%$ sintomas ansiosos.

Piores respostas psicológicas estiveram relacionadas ao histórico de doenças crônicas em australianos $^{23}$ e em italianos ${ }^{31}$. No Brasil ${ }^{22}$, o fato de pertencer ao grupo de risco foi associado a uma pior saúde mental. Na China ${ }^{20}$, no Egito $^{29}$, na Itália ${ }^{12}$, na Jordânia ${ }^{25}$ e na Turquia $^{\mathbf{2 8}}$, maiores prevalências de sintomas depressivos ou ansiosos estiveram associadas à presença de doença crônica prévia.

Problema de saúde mental pré-existente foi um fator preditivo positivo para piores respostas psicológicas na Austrália ${ }^{23}$, no Brasil13,22, no Chipre ${ }^{19}$ e na Itália ${ }^{31}$. Na Bósnia e Herzegovina ${ }^{9}$, indivíduos com diagnóstico prévio de depressão apresentaram maiores níveis de medo.

Na Austrália ${ }^{15}$, na China ${ }^{20}$, no Chipre ${ }^{19}$ e na Coreia do Sul16, mudanças no comportamento de saúde com prejuízo da qualidade de vida estiveram associadas a uma maior prevalência de sintomas depressivos e ansiosos durante a pandemia de Covid-19. Foi identificada diminuição da prática de atividade física, assim como alteração na rotina de sono e no consumo de álcool e tabaco, durante a pandemia de Covid-19. Mulheres, jovens, solteiras, com baixa escolaridade e menor renda foram as mais afetadas. No estudo coreano ${ }^{16}$, embora homens tivessem menos comportamentos preventivos à saúde, ainda apresentaram melhores resultados psicológicos quando comparados às mulheres. 
Estudo realizado nos Estados Unidos avaliou o consumo de tabaco na população durante a pandemia de Covid-19. Foi verificado um aumento de $40 \%$ no consumo e maior percepção do risco da Covid-19 esteve associada a uma maior intenção de cessar o uso ${ }^{\mathbf{2 7}}$.

Estudo americano identificou que, durante a pandemia de Covid-19, houve maior sofrimento psicológico associado ao uso excessivo de álcool e ao número de episódios de uso desta substância, sendo tal associação significativa para as mulheres ${ }^{10}$.

Estudo na China encontrou uma prevalência de uso nocivo de álcool em $11,1 \%$ de seu público-alvo. O aumento do consumo se deu especialmente entre os jovens (21 a 40 anos). Segundo os autores, essa faixa etária estaria psicologicamente mais vulnerável por acessar com frequência informações sobre a Covid-19 nas mídias sociais e também em virtude do bloqueio e do isolamento social experienciados durante a pandemia. No estudo chinês, não foram verificadas diferenças significativas entre gênero e aumento do consumo de álcool36.

\section{Fatores de proteção em saúde mental}

Os estudos analisados identificaram alguns fatores que podem estar relacionados a uma maior proteção à saúde mental dos indivíduos expostos a pandemias como a de Covid-19. Os fatores de proteção mais mencionados nos estudos foram: conhecimento das medidas de prevenção e controle da Covid-19, acesso a informações precisas e técnicas a respeito do vírus, idade avançada, disponibilidade de EPIs e maior

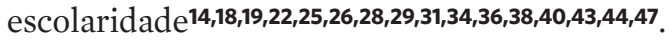

Quanto às características relaciona das à forma de enfrentamento, algumas atitudes e comportamentos foram apontados como fatores de proteção, nos estudos, tais como: boa autopercepção de saúde ${ }^{14}$, adoção de comportamentos positivos relacionados à saúde ${ }^{15,16}$, atitude positiva e otimista em relação à pandemia ${ }^{25,49}$, adoção de estratégias de enfrentamento ativas ${ }^{39}$, comportamentos de enfrentamento cognitivos e pró-sociais ${ }^{\mathbf{1 8}}$, enfrentamento focado no proble$\mathrm{ma}^{14}$, apoio social percebido ${ }^{29,39}$ e participação em atividades sociais e coletivas ${ }^{16}$.

Variáveis sociodemográficas foram apontadas como possíveis fatores de proteção, nos artigos, tais como: residir em ambiente rural9,29, aumento da idade ${ }^{12,25,29}$, ser aposentado $^{25}$, ser casado ou residir com cônjuge ${ }^{\mathbf{1 2}}$, residir com crianças ou menores de 16 anos $^{14}$, maior escolaridade $\mathbf{2 3 , 2 8 , 3 4}^{2}$ estar empregado ${ }^{11,12,47}$ e não ser profissional da saúde 22,40 .

Indivíduos que estavam empregados e não passavam por dificuldades financeiras relataram maior sensação de segurança e menor risco de apresentarem sintomas psiquiátricos/ psicopatologias 28 .

O nível educacional elevado parece estar relacionado à posse de maiores quantidades de informação, a melhores respostas e atitudes em relação à Covid-1928. Nos estudos analisados, foram considerados fatores de proteção em saúde mental: maior conhecimento a respeito da Covid-19, como orientações de especialistas e autoridades em saúde pública, e acesso a informações técnicas ${ }^{\mathbf{2 6}, 30}$, além de menor exposição às mídias sociais ${ }^{\mathbf{1 4}, \mathbf{4 8}}$. Além disso, mais conhecimentos sobre medidas de prevenção e controle da Covid-1925,49 e mais satisfação com as informações de saúde ${ }^{32}$ estiveram relacionados a uma maior a sensação de segurança da população.

A adoção de medidas de precaução, a dis-

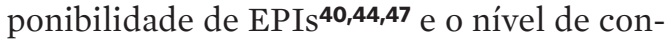
fiança nos profissionais da saúde 32 também constituíram fatores de proteção, nos artigos analisados. A disponibilidade de EPIs em quantidades e qualidades adequadas estaria associada a uma maior percepção de segurança dos profissionais da saúde, e também maior proteção para suas famílias, o que caracteriza um fator preditivo negativo para o sofrimento psicológico destes profissionais ${ }^{\mathbf{2 8}}$.

\section{Intervenções em saúde mental}

Algumas possíveis intervenções em saúde mental para situações de pandemias são 
sugeridas nos estudos analisados. As estratégias são focadas em intervenções voltadas para os profissionais da saúde $35,37,38,41,42,44,45,47$ e para a população em geral20,22,25,26,32-34,36,38,42,47,49.

\section{Intervenções em saúde mental para os profissionais da saúde}

Intervenções em saúde mental voltadas para profissionais da saúde e suas famílias devem estar disponíveis ${ }^{47}$. Para isto, estes profissionais precisam ser incorporados às equipes de saúde e recursos digitais podem ser utilizados, como serviços de atendimento psicológico on-line ${ }^{35,37}$.

As intervenções psicológicas podem auxiliar os profissionais da saúde a lidarem com a pressão, promovendo-lhes bem-estar psicológico, o que pode melhorar sua eficácia no trabalho, a qualidade dos serviços e a segurança dos pacientes ${ }^{44}$. Profissionais da saúde com diagnósticos prévios de problemas de saúde mental devem ser considerados grupos prioritários para essas intervenções ${ }^{37}$.

Alguns recursos importantes podem ser fornecidos aos profissionais da saúde, tais como: apoio social para eles mesmos e para suas famílias, provisionamento de tempo de lazer adequado e melhorias na infraestrutura da instituição. É fundamental garantir-lhes boas condições de trabalho, com equipe suficiente e redução na demanda e na jornada, além de acomodações e locais de descanso apropriados, bem como acesso a EPIs em quantidade e qualidade suficientes $\mathbf{4 2 , 4 7}^{\mathbf{2}}$.

Ademais, outras estratégias podem ser utilizadas para promover a melhoria da saúde mental dos profissionais, como o reconhecimento do trabalho dos que atuam na linha de frente, através de incentivos e promoções ${ }^{44}$. Outra ação que pode ser implementada é o trabalho em equipes mistas, mesclando profissionais mais e menos experientes, o que aumentaria a habilidade prática dos profissionais médicos e diminuiria a sensação de insegurança ${ }^{44}$.

As instituições de saúde podem buscar promover campanhas educacionais, com a divulgação de informações e suporte emocional, ampliando o conhecimento das equipes. Isso poderia minimizar os níveis de ansiedade e estresse, e preparar os profissionais da saúde para situações de pandemias ${ }^{41}$.

Para maior aderência dos profissionais da saúde aos serviços de apoio é necessário desmistificar sentimentos de angústia nas equipes, minimizando o estigma relacionado à saúde mental presente na sociedade e fomentando discussões a respeito dos fatores que causam sofrimento mental nos profissionais. Tal medida buscaria tornar normal a presença de sentimentos como medo e insegurança em profissionais da saúde, pois este público é relutante em reconhecer suas próprias vulnerabilidades, principalmente frente aos seus pares e supervisores ${ }^{\mathbf{3 8}}$.

Estudo com médicos residentes norte-americanos verificou que os profissionais que atuavam na linha de frente no combate à Covid-19 tinham conhecimento dos recursos de apoio psicológico disponíveis na instituição em que atuavam, contudo, apenas $4 \%$ utilizavam tais serviços. Para facilitar a adesão dos profissionais da saúde aos programas de apoio psicológico, as instituições podem disponibilizar os recursos de forma anônima, além de manterem os programas já existentes na instituição $0^{38}$.

Programas que favoreçam melhores condições físicas, mentais e sociais nas equipes médicas, como intervenções individuais e coletivas, partilha de histórias e experiências, são estratégias que podem reforçar atitudes positivas nestes profissionais ${ }^{35}$. Entre as intervenções possíveis, atividades como o Balint Groups podem ajudar profissionais médicos a lidarem com emoções desafiadoras, garantindo-lhes mais qualidade no tratamento dos pacientes ${ }^{44}$. Outra estratégia é o uso da terapia cognitivo-comportamental17,47.

\section{Intervenções em saúde mental para a população geral}

As intervenções em atenção primária devem ser voltadas à prevenção e podem fazer uso da 
comunicação em saúde pública, envolvendo campanhas, ações em saúde e uso de mídias sociais. Informações claras e de fácil compreensão a respeito de cuidados, prevenção, contágio e saúde mental devem ser amplamente divulgadas. Nas mídias sociais, governos podem utilizar perfis oficiais de especialistas em saúde pública para a divulgação de informações oportunas e confiáveis para a população, além de buscar estratégias que minimizem a disseminação de fake news. $\mathrm{O}$ acesso da população a informações de qualidade pode neutralizar os efeitos psicológicos negativos da pandemia, transmitindo maior sensação de segurança e contribuindo para comportamentos de enfrentamento e prevenção, bem como maior sensação de controle 22,26,34,36,38,47.

Os governos e as autoridades de saúde devem garantir que a população tenha acesso a quantidades adequadas de EPIs, produtos de higiene pessoal, serviços e mercadorias essenciais ${ }^{32,49}$. Outro ponto importante a considerar é que alguns indivíduos sofrem perdas econômicas em razão de pandemias, sendo fundamental a elaboração de políticas públicas e a liberação de benefícios, como auxílio financeiro a ser utilizado nesse período ${ }^{22,32}$. Além disto, é necessário aumentar o número de prestadores de serviços psicológicos e sociais que atendam de forma gratuita às demandas de saúde mental da população 22,26 .

Populações mais vulneráveis devem ser priorizadas e ter acesso facilitado a serviços de aconselhamento psicológico ou psiquiátrico. Deve-se fazer um rastreio na população, a fim de identificar indivíduos com condições de saúde mental pré-existentes ou com a presença de sintomatologias psiquiátricas, para possibilitar-lhes o acesso a intervenções em saúde mental com celeridade. Pacientes diagnosticados com Covid-19 e seus familiares também devem ser considerados como grupos prioritários ${ }^{20,33,36}$.

A abordagem a esses indivíduos pode ser realizada por meio de uma equipe multidisciplinar de saúde mental, utilizando plataformas de aconselhamento on-line, através de intervenções digitais, em função de sua praticidade e economicidade, garantindo-lhes o acesso a serviços de saúde mental e tratamentos psiquiátricos ${ }^{20,23,36,42}$. Outras estratégias que podem ser utilizadas: atendimento psicológico por telefone, práticas integrativas e complementares, e exercícios de relaxamento ${ }^{45}$.

Entre as estratégias que o próprio indivíduo pode adotar, incluem-se as limitações do tempo e do tipo de informação que acessa em mídias e redes sociais, sobre a Covid-19, sendo recomendado o tempo máximo de duas horas por dia, concentrando-se apenas em informações necessárias (fatos e dados), evitando tanto o excesso de informações quanto as fake news ${ }^{26,33}$. Além disto, deve-se evitar o acesso a este tipo de informação antes de dormir ${ }^{33}$. Também é importante que o indivíduo mantenha um ritmo normal de trabalho, descanse o máximo possível, pratique exercícios regularmente e tenha uma boa qualidade de sono $^{33}$.

Em períodos de pandemias, se faz ainda mais necessário o ato de prestar atenção às mudanças psicológicas em crianças e jovens. Atendimentos psicológicos on-line especialmente voltados para este público podem ser disponibilizados pelos governos. Além disto, professores que acompanham este alunado têm importante papel, pois, no caso de perceberem quaisquer alterações psicológicas, devem informa-las aos pais/ responsáveis, para que intervenções possam ser realizadas de imediato ${ }^{25}$.

Em alguns casos, a procura por serviços de saúde mental não é a estratégia priorizada para lidar com o sofrimento. Alguns indivíduos podem recorrer a ações autodestrutivas, que causam a sensação de alívio do problema apenas em curto prazo, como o excesso de alimentação, drogas ou álcool22. Portanto, é importante que os serviços de saúde mental estejam preparados para auxiliar indivíduos em situação de uso nocivo ou abuso de substâncias lícitas e ilícitas, para lidar com os quadros de compulsão que possam ter sido desencadeados durante a pandemia de Covid-19, ou 
ainda, com as situações pré-existentes que se acentuem nesse período de crise.

No inicio de uma pandemia, pode haver aumento do apoio social, o que pode atrasar o aparecimento de sintomas de sofrimento psicológico em alguns indivíduos. Contudo, à medida que a coesão social diminui, sintomas de sofrimento, como ansiedade e trauma, podem vir a se manifestar. Por este motivo, recomenda-se que intervenções psicológicas sejam disponibilizadas de imediato e sustentadas em longo prazo, a fim de garantirem um cuidado integral e que vise à recuperação desses indivíduos ${ }^{36}$.

\section{Considerações finais}

Os resultados do presente estudo referem-se à experiência mundial do primeiro ano de pandemia e direcionam um perfil populacional cuja saúde mental está mais vulnerável à Covid-19. Os sintomas psiquiátricos e as psicopatologias mais relatadas nos artigos foram sintomas depressivos, ansiosos, estresse e insônia. Estiveram mais suscetíveis ao impacto psicológico da pandemia de Covid-19: profissionais da saúde, adultos jovens, mulheres; pessoas com menor escolaridade e renda, com histórico de doença crônica ou de transtornos psiquiátricos, e com maior exposição às mídias. A fim de minimizar os impactos negativos da pandemia, intervenções psicológicas devem ser disponibilizadas à população, inclusive de forma on-line. Além disto, é fundamental a presença de profissionais da saúde mental na composição das equipes de saúde, para que possam assegurar apoio psicológico, especialmente aos profissionais que atuam na linha de frente. Ademais, fatores de proteção destacados no estudo podem ser explorados para reduzir os prejuízos na saúde mental dos indivíduos e das coletividades.

Por fim, não se pode deixar de destacar que, embora esta revisão integrativa da literatura apresente uma série de informações relevantes, que podem contribuir para um maior conhecimento acerca do tema saúde mental e Covid-19, os resultados aqui apresentados são de difícil comparação, devido às particularidades dos estudos analisados. Além disto, novos estudos acerca do tema são necessários. Os artigos incluídos nesta revisão são provenientes de diferentes países, com realidades e características específicas e cujas mensurações utilizaram medidas e instrumentos diversos. Outra limitação do presente estudo é a impossibilidade de acompanhar a evolução diária das inúmeras publicações sobre o tema, uma vez que se trata de assunto atual e pouco conhecido, e que novos artigos vêm sendo publicados diariamente.

\section{Colaboradoras}

Lobo LAC (0000-0002-6758-3269)* contribuiu para a concepção, o planejamento, a elaboração, a análise e a interpretação dos resultados, e aprovação da versão final do manuscrito. Rieth CE (0000-0001-9865-1650)* contribuiu para o planejamento da pesquisa e a revisão crítica do trabalho, e aprovação da versão final do manuscrito. 


\section{Referências}

1. Organização Pan-Americana da Saúde. Considerações psicossociais e de saúde mental durante o surto de COVID-19. Geneca: OPAS; 2020.

2. Barros-Delben B, Cruz RM, Trevisan KRR, et al. Saúde mental em situação de emergência: COVID-19. RDP. 2020; 10(2):18-28.

3. Inter-Agency Standing Committee. Guia Preliminar Como lidar com os aspectos psicossociais e de saúde mental referentes ao surto de Covid-19. Versão 1.5. Genebra:IASC; 2020.

4. Organização Pan-Americana da Saúde. Proteção da Saúde Mental em Situações de Epidemias. 2006. [acesso em 2020 abr 1]. Disponível em: https://www.paho. org/hq/dmdocuments/2009/Protecao-da-Saude-Mental-em-Situaciones-de-Epidemias--Portugues. pdf.

5. Brehmer LCF, Trindade LL, Ramos FRS, et al. Revisão integrativa da literatura sobre a Influenza AHıN1. Texto Contexto Enferm. 2011; (11):272-277.

6. Mendes KDS, Silveira RCCP, Galvão CM. Revisão integrativa: método de pesquisa para a incorporação de evidências na saúde e na enfermagem. Texto Contexto Enferm. 2008; 17(4):758-764.

7. Ursi ES, Galvão CM. Prevenção de lesões de pele no período perioperatório: revisão integrativa da literatura. Rev. Latino-am Enfermagem. 2006; 14(1):12431.

8. Li X, Lianting H, Huang T, et al. Factors Associated with Mental Health Results among Workers with Income Losses Exposed to COVID-19 in China. Int. J. Environ. Res. Public Health. 2020; (17):5627.

9. Sljivo A, Kacamakovic M, Quraishi I, et al. Fear and Depression among Residents of Bosnia and Herzegovina during COVID-19 Outbreak - Internet Survey. Psychiatr. Danub. 2020; 32(2):266-272.

10. Rodriguez LM, Litt DM, Stewart SH. Drinking to cope with the pandemic: The unique associations of COVID-19-related perceived threat and psychological distress to drinking behaviors in American men and women. Addictive Behaviors. 2020; (110):106532.

11. Shi L, Zheng-An L, Que JY, et al. Prevalence of and Risk Factors Associated With Mental Health Symptoms Among the General Population in China During the Coronavirus Disease 2019 Pandemic. JAMA Network Open. 2020; 3(7):e2014053.

12. Gualano MR, Lo Moro G, Voglino G, et al. Effects of Covid-19 Lockdown on Mental Health and Sleep Disturbances in Italy. Int. J. Environ. Res. Public Health. 2020; (17):4779.

13. Barros MBA, Lima MG, Szwarcwald CL, et al. Relato de tristeza/depressão, nervosismo/ansiedade e problemas de sono na população adulta brasileira durante a pandemia de COVID 19. Epidemiol. Serv. Saúde. 2020; 29(4):e2020427.

14. Salgado JG, Andrés-Villas M, Domínguez-Salas S, et al. Related Health Factors of Psychological Distress During the COVID-19 Pandemic in Spain. Int. J. Environ. Res. Public Health. 2020; (17):3947.

15. Stanton R, To QG, Khalesi S, et al. Depression, Anxiety and Stress during COVID-19: Associations with Changes in Physical Activity, Sleep, Tobacco and Alcohol Use in Australian Adults. Int. J. Environ. Res. Public Health. 2020; (17):4065.

16. Kim YJ, Cho JH. Correlation between Preventive Health Behaviors and Psycho-Social Health Based on the Leisure Activities of South Koreans in the COVID-19 Crisis. Int. J. Environ. Res. Public Health. 2020; (17):4066.

17. Guo J, Feng XL, Wang XH, et al. Coping with COVID-19: Exposure to COVID-19 and Negative Impact on Livelihood Predict Elevated Mental Health Problems in Chinese Adults. Int. J. Environ. Res. Public Health. 2020; (17):3857. 
18. Antunes R, Frontini R, Amaro N, et al. Exploring Lifestyle Habits, Physical Activity, Anxiety and Basic Psychological Needs in a Sample of Portuguese Adults during COVID-19. Int. J. Environ. Res. Public Health. 2020; (17):4360

19. Solomou I, Constantinidou F. Prevalence and Predictors of Anxiety and Depression Symptoms during the COVID-19 Pandemic and Compliance with Precautionary Measures: Age and Sex Matter. Int. J. Environ Res Public Health. 2020; 17(14):4924.

20. Guo Y, Cheng C, Zeng Y, et al. Mental Health Disorders and Associated Risk Factors in Quarantined Adults During the COVID-19 Outbreak in China: Cross-Sectional Study. J. Med. Internet Res. 2020; 22(8):e20328.

21. Taylor S, Landry CA, Paluszek MM, et al. COVID stress syndrome: Concept, structure, and correlates. Depression and anxiety. 2020; 37(8):706-714.

22. Duarte M Q, Portella GJ, Marceli CT. COVID-19 e os impactos na saúde mental: uma amostra do Rio Grande do Sul, Brasil. Ciênc. Saúde Colet. 2020; 25(9):34013411.

23. O'Moore K, Tang S, Christensen $\mathrm{H}$, et al. Acute mental health responses during the COVID-19 pandemic in Australia. PloSONE. 2020; 15(7):e0236562.

24. Pedrozo-Pupo JC, Pedrozo-Cortés MJ, Campo-Arias A. Perceived stress associated with COVID-19 epidemic in Colombia: an online survey. Cad. Saúde Pública. 2020; 36(5):e00090520.

25. Naser AY, Dahmash EZ, Rabaa AR, et al. Mental health status of the general population, healthcare professionals, and university students during 2019 coronavirus disease outbreak in Jordan: A cross-sectional study. Brain Behav. 2020; (10):e0173.

26. Chao M, Xue D, Liu T, et al. Media use and acute psychological outcomes during COVID-19 outbreak in China. J. Anxiety Disord. 2020; 74:102248.

27. Kowitt SD, Cornacchione RJ, Jarman KL, et al. To- bacco Quit Intentions and Behaviors among Cigar Smokers in the United States in Response to COVID-19. Int. J. Environ. Res. Public Health. 2020; 17(15):5368.

28. Özdin S, Bayrak Özdin Ş. Levels and predictors of anxiety, depression and health anxiety during COVID-19 pandemic in Turkish society: The importance of gender. Int. J. Soc. Psychiatry. 2020; 66(5):504511.

29. El-Zoghby SM, Soltan EM, Salama HM. Impact of the COVID-19 Pandemic On Mental Health and Social Support Among Adult Egyptians. Journal of Community Health. 2020; 45(4):689-695.

30. Roy D, Tripathy S, Kar SK, et al. Study of knowledge, attitude, anxiety \& perceived mental healthcare need in Indian population during COVID-19 pandemic. Asian J. Psychiatr. 2020; (51):102083.

31. Mazza C, Ricci E, Biondi S, et al. Nationwide Survey of Psychological Distress among Italian People during the COVID-19 Pandemic: Immediate Psychological Responses and Associated Factors. Int. J. Environ. Res. Public Health. 2020; 17(9):3165.

32. Wang C, Pan R, Wan X, et al. A longitudinal study on the mental health of general population during the COVID- 19 epidemic in China. Brain Behav. Immun. 2020; (87):40-48.

33. Huang Y, Zhao N. Generalized anxiety disorder, depressive symptoms and sleep quality during COVID-19 outbreak in China: a web-based cross-sectional survey. Psychiatry Res. 2020; (288):112954.

34. Tian F, Li H, Tian S, et al. Psychological symptoms of ordinary Chinese citizens based on SCL-90 during the level I emergency response to COVID-19. Psychiatry Res. 2020; (288):112992.

35. Zhang W, Wang K, Yin L, et al. Mental Health and Psychosocial Problems of Medical Health Workers during the COVID-19 Epidemic in China. Psychother. Psychosom. 2020; 89(4):242-250. 
36. Ahmed MZ, Ahmed O, Aibao Z, et al. Epidemic of COVID-19 in China and associated Psychological Problems. Asian Journal of Psychiatry. 2020; (51):1020922.

37. Cai Q, Feng H, Huang J, et al. The mental health of frontline and non-frontline medical workers during the coronavirus disease 2019 (COVID-19) outbreak in China: A case-control study. J. Affect. Disor. 2020; (275):210-215.

38. Kannampallil TG, Goss CW, Evanoff BA, et al. Exposure to COVID-19 patients increases physician trainee stress and burnout. PLoS ONE. 2020; 15(8):e0237301.

39. Si M, Su XY, Jiang Y, et al. Psychological impact of COVID-19 on medical care workers in China. Infectious Diseases of Poverty. 2020; 9(113):1.

40. Moreno LL, Talavera BV, Albuerne YG, et al. Symptoms of Posttraumatic Stress, Anxiety, Depression, Levels of Resilience and Burnout in Spanish Health Personnel during the COVID-19 Pandemic. Int. J. Environ. Res. Public Health. 2020; (17):5514.

41. Temsah MH, Al-Sohime F, Alamro N, et al. The psychological impact of COVID-19 pandemic on health care workers in a MERS-CoV endemic country. J. Infect. Pub. Health. 2020; (13):877-882.

42. Zerbini G, Ebigbo A, Reicherts P, et al. Psychosocial burden of healthcare professionals in times of COVID-19 - a survey conducted at the University Hospital Augsburg. Ger. Med. Sci. 2020; (18):Doc05.

43. Man MA, Toma C, Motoc NS, et al. Disease Perception and Coping with Emotional Distress During COVID-19 Pandemic: A Survey Among Medical Staff. Int. J. Environ Res. Public Health. 2020; 17(13):4899.

44. Xiao X, Zhu X, Fu S, et al. Psychological impact of healthcare workers in China during COVID-19 pneu- monia epidemic: A multi-center cross-sectional survey investigation. J. Affect. Disord. 2020; (274):405410.

45. Dal'Bosco EB, Fiorino LSM, Skupien SV, et al. A saúde mental da enfermagem no enfrentamento da COVID-19 em um hospital universitário regional. Revista Brasileira de Enfermagem. 2020; 73(2):1-7.

46. Lai J, Ma S, Wang Y, et al. Factors Associated With Mental Health Outcomes Among Health Care Workers Exposed to Coronavirus Disease 2019. JAMA Netw. Open. 2020; 3(3):e203976.

47. Ying Y, Ruan L, Kong F, et al. Mental health status among family members of health care workers in Ningbo, China, during the coronavirus disease 2019 (COVID-19) outbreak: a cross-sectional study. BMC Psychiatry. 2020; 20(1):379.

48. Duan L, Shao X, Wang Y, et al. An investigation of mental health status of children and adolescents in china during the outbreak of COVID-19. J. Affect. Disord. 2020; 1(275):112-118.

49. Zhou SJ, Zhang LG, Wang LL, et al. Prevalence and socio-demographic correlates of psychological health problems in Chinese adolescents during the outbreak of COVID-19. Eur. Child Adolesc. Psychiatry. 2020; 29(6):749-758.

50. Tang $\mathrm{W}, \mathrm{Hu} \mathrm{T}, \mathrm{Hu} \mathrm{B}$, et al. Prevalence and correlates of PTSD and depressive symptoms one month after the outbreak of the COVID-19 epidemic in a sample of home-quarantined Chinese university students. J. Affect. Disord. 2020; (274):1-7.

\footnotetext{
Recebido em 24/02/2021

Aprovado em 15/05/2021

Conflito de interesses: inexistente

Suporte financeiro: não houve
} 